\title{
COMUNICAÇÃO \\ TÉCNICA DE FISTULAÇÃo E CANULAÇÃo DO RÚMEN EM BOVINOS E OVINOS
}

\author{
Rumen fistulation and cannulation technique in cattle and sheep
}

\author{
Leonardo Augusto Lopes Muzzi', Ruthnéa Aparecida Lázaro Muzzi², Endrigo Leonel Alves Gabellini
}

\begin{abstract}
RESUMO
Foi desenvolvida e descrita uma técnica cirúrgica para fistulação e canulação do rúmen em animais utilizados nos estudos de nutrição animal. O método cirúrgico foi empregado para canulação de 25 vacas e oito cordeiros. A fistulação permanente foi realizada no centro da fossa paralombar esquerda em estágio operatório único. Uma cânula de borracha foi implantada na parede ruminal após o afastamento da musculatura da parede abdominal e a sutura do rúmen ao peritôneo e à pele. Não foram observadas complicações graves advindas da canulação ruminal, mas houve a ocorrência de discreto vazamento de conteúdo em alguns animais. As cânulas permaneceram funcionais por longo período e permitiram a obtenção de quantidades suficientes de amostra.
\end{abstract}

Termos para indexação: Cirurgia, cânula ruminal.

\begin{abstract}
A surgical technique for rumen fistulation and cannulation was developed for animal nutritional studies. The surgical method was used to cannulated 25 adult cows and eight lambs. The permanent fistula was made in the middle of the left paralumbar fossa in a one-stage operation. It was performed the spreading of the abdominal muscle layers and the sutures from the rumen to the peritoneum and to the skin, and the rubber cannula was inserted into the rumen wall. No severe complication was observed in ruminal cannulation, but minor leakages were observed in some animals. The cannulas had a long functional life and permited convenient sampling of digesta.
\end{abstract}

Index terms: Surgery, ruminal cannula.

(Recebido em 10 de janeiro de 2007 e aprovado em 25 de maio de 2007)

A canulação do rúmen em bovinos e ovinos é uma técnica que possui grande importância nos estudos de nutrição animal. Com a implantação de cânulas, é possível avaliar o metabolismo e a fisiologia do pré-estômago (DUFFIELD et al., 2004; GROVUM, 1989; THYFAULT et al., 1975). Nos animais fistulados, também se pode pesquisar a degradabilidade in situ (MUNIZ et al., 2008; SANTRA \& KARIM, 2002). A fistulação do rúmen e a implantação da cânula são realizadas mediante intervenção cirúrgica, que variam conforme o tipo de cânula utilizada. Além disso, a fistulação pode ser realizada em um ou dois estágios operatórios (CATTELAN et al., 1990). Diferentes técnicas cirúrgicas para canulação ruminal foram descritas na literatura (ATASOY \& TAS, 2003; CABRERA et al., 1996; GODWIN \& CHAFFEY, 1988; HORIGANE et al., 1989; MOGHA \& BHARGAVA, 1979).

É possível a obtenção do líquido ruminal através de pequenas cânulas ou sondas inseridas no rúmen (CORLEY III et al., 1999; PASS, 1991), entretanto, a coleta da amostra frequentemente torna-se comprometida em consequencia do entupimento da sonda, além de não permitir a obtenção de grande quantidade do conteúdo fibroso (HORIGANE et al., 1989). Nos ensaios de degradabilidade ou quando é necessária a obtenção de amostras de diferentes locais do rúmen é desejável que sejam instaladas cânulas largas que permitam o fácil acesso ao interior do rúmen (HARRISON, 1995). Existe uma grande variedade de cânulas ruminais, podendo ser preparadas com material rígido, como metal, plástico ou acrílico; ou confeccionadas com material flexível, como borracha, silicone ou vinil. De forma geral, as cânulas fabricadas com material maleável são mais fáceis de serem implantadas e manipuladas (KOMAREK, 1981).

Algumas complicações podem ocorrer após a implantação de cânulas permanentes, como peritonite e vazamento de conteúdo ruminal. $\mathrm{O}$ vazamento de grande quantidade de conteúdo do rúmen pode levar o animal à desidratação e desnutrição (GROVUM, 1989). Além disso, o líquido ruminal extravasado provoca lesões na pele, produz odor desagradável e favorece o aparecimento de miíases. A fistulação ideal é aquela que forma uma vedação em torno da cânula, impedindo o vazamento no período experimental.

'Médico Veterinário, Doutor, Professor Adjunto - Setor de Cirurgia Veterinária/SCV - Departamento de Medicina Veterinária/DMV - Universidade Federal de Lavras/UFLA - Cx. P. 3037 - 37200-000 - Lavras, MG - lalmuzzi@ufla.br

${ }^{2}$ Médica Veterinária, Doutora, Professora Adjunto - Setor de Clínica Médica/SCMPA - Departamento de Medicina Veterinária/DMV - Universidade Federal de Lavras/UFLA - Cx. P. 3037 - 37200-000 - Lavras, MG - ralmuzzi@ufla.br

${ }^{3}$ Médico Veterinário, Professor Substituto - Departamento de Clínica e Cirurgia Veterinária/DCCV - Universidade Federal de Minas Gerais/UFMG Avenida Antônio Carlos 6627 - Campus da UFMG - Cx. P. 567 - 30123-970 - Belo Horizonte, MG - endrigogabellini@yahoo.com.br 
Neste trabalho, objetivou-se desenvolver e descrever o procedimento cirúrgico para fistulação e canulação do rúmen em bovinos e ovinos, realizado em estágio operatório único. Objetivou-se também relatar a evolução pósoperatória dos animais, enfatizando a utilização de técnicas que minimizam a ocorrência de complicações.

As cânulas foram implantadas em animais experimentais das espécies bovina e ovina, no período de 2000 a 2005 . O procedimento cirúrgico foi realizado em 25 vacas adultas, com peso corporal variando entre 340 e $580 \mathrm{~kg}$, sendo 14 da raça Holandesa, sete da raça Girolanda e quatro da raça Jersey. Dos animais da espécie ovina, foram utilizados oito cordeiros da raça Santa Inês, com peso corporal variando entre 30 e $38 \mathrm{~kg}$.

Para implantação no rúmen, foram utilizadas cânulas fabricadas em material de borracha natural maleável produzidas pela Kehl Polímeros Ltda. A cânula para bovinos era constituída por uma peça principal (corpo da cânula) composta por uma aba interna com borda arredondada de 19,0 cm de diâmetro, e uma aba externa plana de 24,0 cm de diâmetro, com distância entre elas de 7,0 cm. A parte central continha uma abertura circular com $11,0 \mathrm{~cm}$ de diâmetro interno e 11,5 cm de diâmetro externo (Figura 1). A tampa da cânula era inserida no orifício central sob pressão. A cânula para ovinos era constituída do mesmo material e apresentava as mesmas estruturas, com menores dimensões. As abas interna e externa possuíam diâmetros de 12,5 e 14,5 cm, com distância entre elas de $3,5 \mathrm{~cm}$. A abertura central apresentava $7,8 \mathrm{~cm}$ de diâmetro interno e $8,3 \mathrm{~cm}$ de diâmetro externo, e a tampa também era encaixada na abertura central sob pressão.

Os bovinos previamente selecionados para o experimento foram submetidos ao jejum alimentar e hídrico de 36 e 12 horas, respectivamente. As vacas foram mantidas em posição quadrupedal por meio de contenção no tronco para

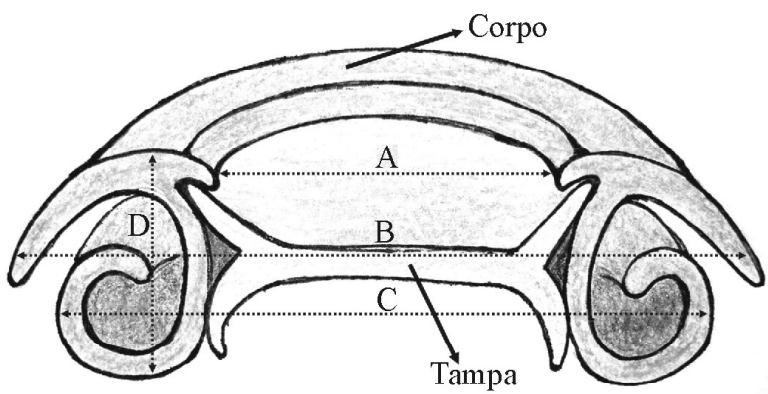

Figura 1 - Representação esquemática da cânula de borracha para implantação no rúmen, em corte transversal. A: diâmetro interno da abertura central; B: diâmetro da aba externa; C: diâmetro da aba interna; D: distância entre as abas. As medidas para bovinos e ovinos são, respectivamente: A: $11,0 \mathrm{~cm}$ e $7,8 \mathrm{~cm} ; \mathrm{B}: 24,0 \mathrm{~cm}$ e $14,5 \mathrm{~cm}$, C: $19,0 \mathrm{~cm}$ e $12,5 \mathrm{~cm}$; D: $7,0 \mathrm{~cm}$ e $3,5 \mathrm{~cm}$. grandes animais, com possibilidade de acesso cirúrgico à região da fossa paralombar esquerda. Realizou-se a tricotomia do flanco esquerdo, iniciando-se na linha média dorsal e se estendendo até a base do úbere. Cada animal foi submetido à sedação com a administração intramuscular de cloridrato de xilazina, na dose de $0,1 \mathrm{mg} / \mathrm{kg}$. Após antissepsia, a fossa paralombar esquerda foi anestesiada por meio do bloqueio neural paralombar, utilizando-se anestesia infiltrativa com cloridrato de lidocaína a $2 \%$. O campo cirúrgico foi isolado e mantido estéril com a utilização de pano de campo fenestrado. Inicialmente, realizou-se uma incisão circular de pele de aproximadamente $12 \mathrm{~cm}$ de diâmetro, iniciando-se $8 \mathrm{~cm}$ ventralmente aos processos transversos das vértebras lombares, mantendo-se o ponto central da circunferência entre a tuberosidade coxal e a $13^{a}$ costela. Toda a porção de pele incisada foi dissecada e removida, formando uma falha circunferencial na pele. Os músculos oblíquo externo, oblíquo interno e transverso do abdome foram divulsionados e afastados no sentido de suas fibras musculares, permitindo acesso ao peritônio, que foi perfurado e incisado com auxílio de tesoura (Figura 2).

Após a abertura da cavidade abdominal, uma porção dorsal do rúmen foi apreendida por duas pinças Backhaus (ou pinças atraumáticas de Glock) e tracionada através da incisão abdominal, expondo parte da parede ruminal. A porção exposta do rúmen foi suturada ao peritônio em torno da abertura cirúrgica, utilizando-se fio catgut cromado $\mathrm{n}^{\circ} 1$ em padrão simples contínuo. Com a parede do rúmen ainda sob tração, quatro suturas separadas de colchoeiro ("U" horizontal) foram aplicadas de forma eqüidistante (uma em cada ponto cardeal) a $1 \mathrm{~cm}$ da borda da incisão de pele, englobando apenas parede do rúmen e pele, utilizando-se fio cirúrgico de nylon monofilamentoso $\mathrm{n}^{\circ} 2$. Entre as suturas de colchoeiro previamente aplicadas, suturas adicionais de colchoeiro foram realizadas, promovendo adequada justaposição entre rúmen e pele, sendo necessário que as suturas ficassem bem próximas umas das outras (Figura 3).

Em seguida, realizou-se a incisão da porção exposta da parede do rúmen, circundando a borda da pele incisada a cerca de $0,5 \mathrm{~cm}$ de distância dos pontos de sutura, promovendo a retirada de um retalho circular da parede do rúmen (Figura 4). A hemorragia da parede ruminal foi controlada por pinçamento e ligadura dos grandes vasos. Procedeu-se a implantação da cânula logo após a formação da fístula ruminal. A cânula foi mantida em água aquecida para torná-la mais flexível. A aba interna foi parcialmente evertida para o interior do orifício central, para facilitar a inserção da cânula no rúmen. A cânula foi inserida na fístula ruminal e a aba interna foi pressionada para o interior do rúmen, permitindo que a cânula adquirisse adequado posicionamento. A aba interna apresentou-se fixa à parede interna do rúmen, enquanto a aba externa da cânula permaneceu justaposta à pele e o tubo central ocupou o espaço criado pela fístula (Figura 5). 


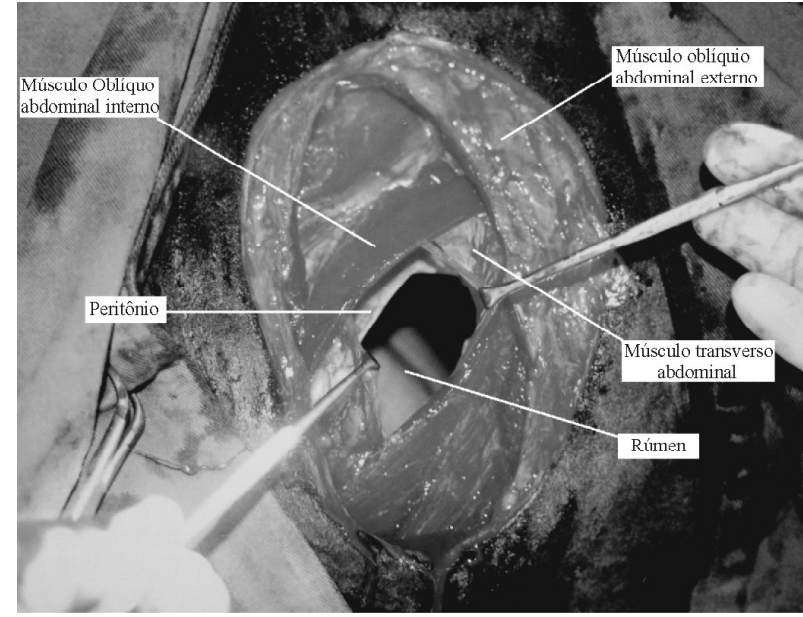

Figura 2 - Abordagem cirúrgica para fistulação ruminal em bovino. No centro da fossa paralombar esquerda realizouse uma incisão circular de pele de aproximadamente $12 \mathrm{~cm}$ de diâmetro. A porção de pele foi dissecada e removida, formando uma falha circunferencial no flanco. Os músculos oblíquo abdominal externo, oblíquo abdominal interno e transverso abdominal foram divulsionados e afastados no sentido de suas fibras. O peritônio foi incisado, permitindo acesso ao saco dorsal do rúmen.

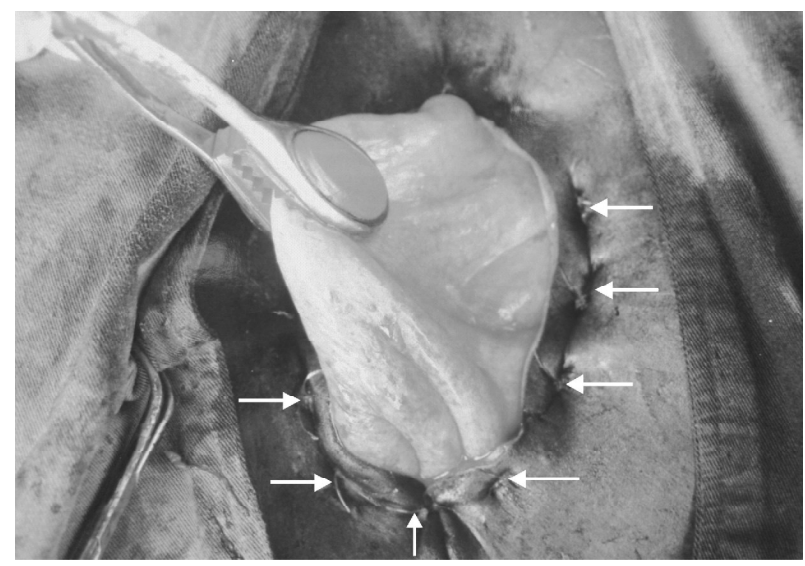

Figura 3 - Técnica cirúrgica para fistulação ruminal em bovino. Uma porção dorsal do rúmen foi apreendida pela pinça de Glock e tracionada através da abertura abdominal. A porção exposta do rúmen foi inicialmente suturada ao peritônio. Com a parede do rúmen sob tração, suturas separadas de colchoeiro ("U” horizontal) foram aplicadas englobando apenas parede do rúmen e pele (setas). É indicado que as suturas de colchoeiro sejam aplicadas próximas entre si.

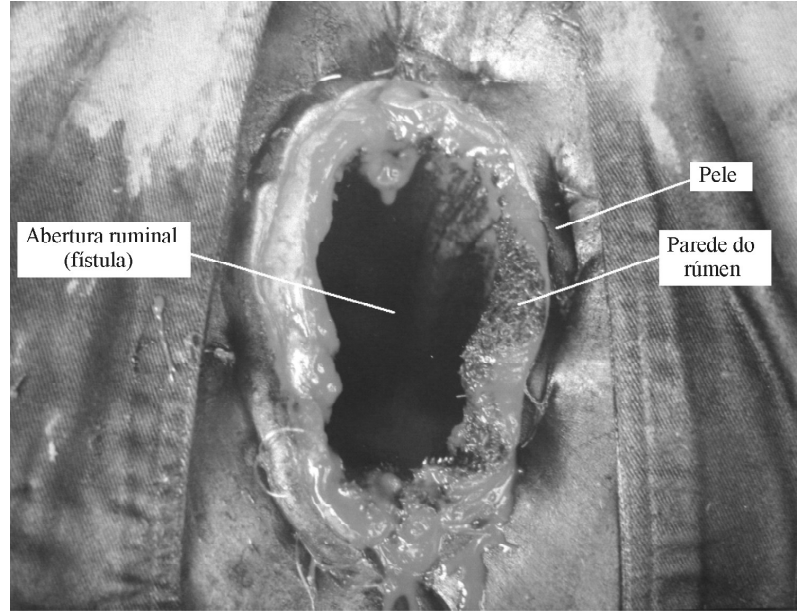

Figura 4 - Técnica cirúrgica para fistulação ruminal em bovino. Após a aplicação das suturas de colchoeiro para adequada justaposição entre parede do rúmen e pele, realizou-se a incisão da porção exposta da parede do rúmen, circundando a borda da pele a cerca de $0,5 \mathrm{~cm}$ de distância dos pontos de sutura. A remoção do retalho circular da parede do rúmen promoveu a formação da fístula ruminal. A hemorragia da parede do rúmen foi controlada por pinçamento e ligadura dos grandes vasos.

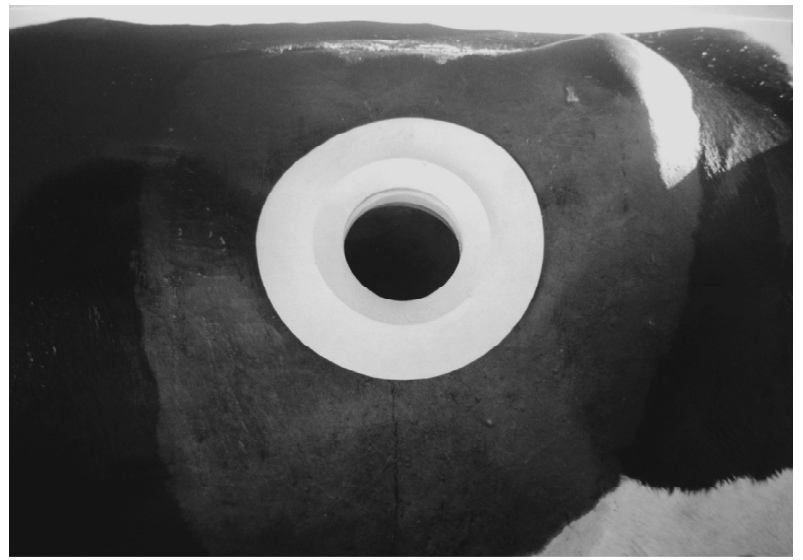

Figura 5 - Canulação do rúmen em bovino. Cânula de borracha recém implantada na fístula ruminal, ainda sem a colocação da tampa. Observar a ampla abertura central e a justaposição da aba externa à pele. A cânula deve posicionar-se na região central do flanco esquerdo.

Nos ovinos, realizou-se a implantação das cânulas com o animal em decúbito lateral, sobre a mesa cirúrgica. Os cordeiros foram mantidos em jejum alimentar e hídrico 
de 36 e 12 horas, respectivamente. Antes da aplicação da xilazina, utilizou-se também como medicação pré-anestésica sulfato de atropina, na dose de $0,1 \mathrm{mg} / \mathrm{kg}$, via intramuscular. A fossa paralombar esquerda foi anestesiada por meio de bloqueio anestésico em " $\mathrm{L}$ " invertido, utilizando cloridrato de lidocaína a $2 \%$. O retalho circunferencial de pele removido da parede do flanco apresentava diâmetro de 9,0 $\mathrm{cm}$. A técnica cirúrgica utilizada para fistulação e canulação do rúmen foi a mesma descrita para os bovinos, exceto em relação às dimensões da fistula ruminal.

No pós-operatório foi utilizada antibioticoterapia preventiva em dose única, sendo administrada penicilina benzatina, na dose de $30.000 \mathrm{UI} / \mathrm{kg}$, via intramuscular. Também foi administrada flunixina meglumina, na dose de $1,1 \mathrm{mg} / \mathrm{kg}$, via intramuscular, a cada 24 horas, durante três dias. Realizou-se a limpeza diária da ferida cirúrgica com solução antisséptica de clorexidina a $0,5 \%$, seguida da aplicação de pomada antibiótica e cicatrizante (Vetaglós ${ }^{\circledR}$ ). As suturas de náilon foram removidas em 14 dias. Os animais retornaram à alimentação normal 24 horas após a intervenção e estavam aptos aos ensaios nutricionais três semanas após a cirurgia.

A contenção dos bovinos em posição quadrupedal e dos ovinos em decúbito lateral, e os tipos de sedação e anestesia utilizados, propiciaram a adequada realização da fistulação do rúmen, aspectos também descritos por Cabrera et al. (1996), Cattelan et al. (1990) e Mcsweeney (1989). Pelo fato de os cordeiros terem sido operados em decúbito, a atropina foi administrada com o objetivo de reduzir a salivação e a motilidade do rúmen (KOMAREK, 1981). Deve-se ter cautela ao operar os ovinos em decúbito lateral, pois o rúmen exerce pressão sobre a cavidade abdominal, podendo alterar os planos anatômicos. Além disso, há uma maior possibilidade de contaminação da ferida cirúrgica após a abertura do rúmen.

Nas duas primeiras semanas de pós-operatório, a borda da pele suturada ao rúmen apresentou discreta necrose tecidual em $80 \%$ dos animais. Essa delgada borda necrosada foi manualmente removida, sendo substituída por tecido de granulação, e não promoveu o afrouxamento da cânula. Segundo Ducharme (1990) e Mcsweeney (1989) é comum a ocorrência de necrose na borda da ferida cirúrgica em consequência da compressão dos tecidos exercida pela cânula, e a ferida geralmente se recupera com cicatrização por segunda intenção. É importante destacar que mesmo ocorrendo esta necrose na borda da pele, não é recomendada a ampliação da incisão circular de pele, pois isso assegura a estabilidade da cânula. Neste estudo, cerca de 30 dias após a intervenção, houve adequada cicatrização da ferida cirúrgica, havendo continuidade entre pele e mucosa do rúmen.

Não ocorreu edema acentuado em torno das cânulas ou enfisema subcutâneo. Enfisema subcutâneo foi observado por Cattelan et al. (1990) em 62\% dos bovinos canulados, mas sem acarretar problemas na cicatrização. Acredita-se que a perfeita aposição entre pele e rúmen promovida pelas suturas de colchoeiro tenha impedido a formação do enfisema subcutâneo. Não é comum a ocorrência de complicações graves advindas da canulação do rúmen (ATASOY \& TAS, 2003; MCSWEENEY, 1989), e na atual pesquisa não se observou qualquer caso clínico de peritonite. A ausência de casos de peritonite pode ser justificada pela sutura do rúmen ao peritônio, que impediu a queda de conteúdo ruminal na cavidade abdominal.

Dos 25 bovinos submetidos à canulação do rúmen, houve discreto vazamento de conteúdo ruminal entre a cânula e a fístula em seis animais (24\%). Em um desses animais, o vazamento se intensificou após seis meses da intervenção cirúrgica, sendo retirado dos experimentos de digestibilidade. Entre os ovinos operados, dois apresentaram vazamento de conteúdo ruminal (25\%). Em um deles, houve expulsão da cânula na primeira semana após a cirurgia, sendo retirado dos experimentos. $\mathrm{O}$ vazamento de conteúdo ruminal é uma das complicações mais comumente observadas em animais canulados (CABRERA et al., 1996; GROVUM, 1989; KOMAREK, 1981; SANTRA \& KARIM, 2002; THYFAULT et al., 1975). O estudo de Grovum (1989) descreve a utilização de dispositivos acoplados à cânula com a finalidade de reduzir o vazamento do conteúdo ruminal, entretanto, ainda foram observados índices de $20 \%$ de vazamento em ovinos.

No atual estudo, a musculatura da parede abdominal não foi incisada, mas divulsionada no sentido das fibras musculares, proporcionando uma abertura muscular em "grade". Após a implantação da cânula, essa musculatura passou a atuar como um esfíncter, retendo e comprimindo a parte central da cânula na abertura abdominal. A utilização dessa técnica auxilia na formação de uma vedação natural em torno da cânula, diminuindo o vazamento do conteúdo ruminal (DUCHARME, 1990; NOORDSY, 1994). No cordeiro em que houve a expulsão da cânula, não foi observada abertura anormal da fístula ou cicatrização irregular da ferida. Entretanto, observou-se que a fístulação ruminal foi realizada na altura do sulco longitudinal esquerdo, que divide o rúmen em saco dorsal e saco ventral. Nesse ponto, o rúmen possui uma elevação tecidual que impeliu a aba interna da cânula para o exterior, expulsando-a. Portanto, recomenda-se ter o cuidado de realizar a fistulação e implantação da cânula em ovinos somente no saco dorsal do rúmen. 
Os procedimentos cirúrgicos para fistulação e canulação do rúmen duraram, em média, 50 minutos por animal. A técnica cirúrgica descrita apresenta as particularidades discutidas que tendem a aumentar o tempo operatório, mas devem ser seguidas para que o resultado seja satisfatório. Apesar disso, a técnica descreve a implantação da cânula em apenas um tempo operatório. Cattelan et al. (1990) e Harrison (1995) relatam a canulação do rúmen em dois estágios operatórios, com intervalo de oito dias entre eles. O método de implantação da cânula em um único estágio mostrou-se eficaz, reduzindo o custo operacional e o estresse do animal.

Foram utilizadas cânulas flexíveis de borracha, mas acredita-se que a técnica cirúrgica descrita também possa ser utilizada para a implantação de cânulas ruminais rígidas. A cânula utilizada apresentava um grande diâmetro interno, o que permitiu a coleta de grandes quantidades de conteúdo ruminal com facilidade. Além disso, a cânula não se apresentava protuberante sobre a região do flanco, permitindo que os animais permanecessem em condições de pastagem sem a perda do equipamento. Segundo Komarek (1981) e Santra \& Karim (2002) os animais fistulados podem ser mantidos por longos períodos nos ensaios experimentais, desde que sejam tomados os cuidados rotineiros de limpeza e higiene do local. Nos relatos de Godwin \& Chaffey (1988) e Horigane et al. (1989), os animais permaneceram canulados sem maiores complicações por períodos de três e quatro anos, respectivamente. No estudo atual, quatro vacas encontravam-se canuladas há cinco anos, sem demonstrar alterações clínicas e sem apresentar deterioração da cânula.

A técnica cirúrgica descrita para fistulação do rúmen e implantação de cânula mostra-se eficaz e com mínimas complicações pós-operatórias. A utilização do método em estágio operatório único reduz o custo operacional e o estresse do animal, sem alterar a eficácia do procedimento. Para reduzir o índice de complicações pós-operatórias é importante a utilização da técnica de afastamento das fibras musculares da parede abdominal e sutura do rúmen ao peritônio e à pele. As cânulas de borracha permanecem funcionais por longo período e permitem a obtenção de quantidades suficientes de amostra.

\section{REFERÊNCIAS BIBLIOGRÁFICAS}

ATASOY, N.; TAS, A. Considerations for gastrointestinal cannulation (rumen, duodenum and ileum) in sheep with a ruminal, a simple t-type and a modified t-type cannula. Deutsche Tierärztliche Wochenschrift, Hannover, v. 110, n. 7, p. 299-302, 2003.
CABRERA, C. R.; LOPEZ, C. A.; MAIZTEGUI, V. J.; MARIN, G. M. P. Fistulacion y canulacion permanente del compartimento 1 (rumen) en alpacas. Avances en Ciencias Veterinarias, Santiago, v. 11, n. 2, p. 108-111, 1996.

CATTELAN, J. W.; CANOLA, J. C.; VALADÃO, C. A. A.; MACORIS, D. G. Rumenostomia canulada em dois tempos operatórios em bovinos e ovinos. Ars Veterinária, Jaboticabal, v. 6, n. 2, p. 112-119, 1990.

CORLEY III, R. N.; MURPHY, M. R.; LUCENA, J.; PANNO, S. V. A device for obtaining time-integrated samples of ruminal fluid. Journal of Animal Science, Savoy, v. 77, n. 9, p. 2540-2544, 1999.

DUCHARME, N. G. Surgery of the bovine forestomach compartments. The Veterinary Clinics of North America. Food Animal Practice, Philadelphia, v. 6, n. 2, p. 371-397, 1990.

DUFFIELD, T.; PLAIZIER, J. C.; FAIRFIELD, A.; BAGG, R.; VESSIE, G.; DICK, P.; WILSON, J.; ARAMINI, J.; MCBRIDE, B. Comparison of techniques for measurement of rumen $\mathrm{pH}$ in lactating dairy cows. Journal of Dairy Science, Savoy, v. 87, n. 1, p. 59-66, 2004.

GODWIN, I. R.; CHAFFEY, G. A. Simple rapid method of rumen cannulation. Australian Veterinary Journal, Sidney, v. 65, n. 7, p. 227-228, July 1988.

GROVUM, W. L. An improved rumen cannulation technique to minimize leakage. Acta Veterinaria Scandinavica - Supplementum, Londres, v. 86, p. 225228, 1989.

HARRISON, F. A. Surgical techniques in experimental farm animals. Oxford: Oxford University, 1995. 159 p.

HORIGANE, A.; GOCHOU, S.; KISHI, F.; OHTOMO, Y.; HORIGUSHI, M. Silicone rumen cannula with a soft cylindrical part and a hard flange. Journal of Dairy Science, Savoy, v. 72, n. 12, p. 3230-3232, 1989.

KOMAREK, R. J. Rumen and abomasal cannulation of sheep with specially designed cannulas and a cannula insertion instrument. Journal of Animal Science, Savoy, v. 53, n. 3, p. 790-795, 1981. 
MCSWEENEY, C. S. Cannulation of the rumen in catle and buffaloes. Australian Veterinary Journal, Sidney, v. 66, n. 8, p. 266-268, Aug. 1989.

MOGHA, I. V.; BHARGAVA, A. K. Rumen fistula technique and utility. The Indian Veterinary Journal, Madras, v. 56, n. 10, p. 885-887, Oct. 1979.

MUNIZ, J. A.; SAVIAN, T. V.; SCALON, J. D.; Parameters estimation in the model for in situ degradability of mertens and loften. Ciência e Agrotecnologia, Lavras, v.32, n.5, p. 1622-1628, set./out., 2008.
NOORDSY, J. L. Food animal surgery. 3. ed. New Jersey: VLS Books, 1994. 302 p.

PASS, M. A. A stomach tube for the collection of ruminal fluid from sheep. Australian Veterinary Journal, Sidney, v. 68, n. 9, p. 312-313, Sept. 1991.

SANTRA, A.; KARIM, S. A. Rumen cannulation in sheep and goats: fabrication of cannula and surgical procedure for its implantation. Indian Journal of Animal Sciences, Nova Delhi, v. 72, n. 11, p. 978-980, Nov. 2002.

THYFAULT, H. A.; LEFFEL, E. C.; HUANG, M. D. Simplified method for producing permanent ruminal fistulae. Journal of Dairy Science, Savoy, v. 58, n. 12, p. 1899-1901, 1975. 